\title{
Detección de placas de ateroma mediante radiograflas dentales
}

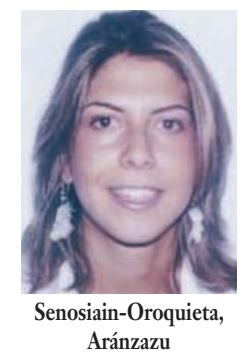

Detection of atheroma plaques by dental radiographs

\section{Senosiain-Oroquieta, Aranzazu* Pardo-López, Berta** \\ De Carlos-Villaffance, Fêlix ${ }^{* * *}$ Cobo-Pland, Juan ${ }^{* * * *}$}

\author{
*Postgrado de Ortodoncia. \\ ${ }^{*}$ Colaboradora de honor. \\ ***Profesor asociado. \\ ****Catedrático de Ortodoncia. \\ Universidad de Oviedo
}

\section{Correspondencia}

\author{
Aránzazu Senosiain Oroquieta \\ Clínica Universitaria de Odontología \\ Universidad de Oviedo \\ C/ Catedrático José Serrano s/n \\ 33006 Oviedo \\ E-mail: senosiaindent@yahoo.es
}

\begin{abstract}
Resumen: Introducción: el accidente cerebrovascular o «stroke» es la tercera causa de muerte en Estados Unidos y representa casi la mitad de las muertes ocurridas cada año en España. Una de las posibles causas del stroke es la formación de placas de ateroma en la bifurcación carotídea. En ocasiones, estas placas se calcifican y pueden ser detectadas de manera casual en la consulta dental mediante una radiografía panorámica o telerradiografía. Material y método: se han revisado las ortopantomografías de 1300 pacientes pertenecientes a la Escuela de Estomatología de la Universidad de Oviedo. Para el estudio, sólo se tuvieron en cuenta los pacientes mayores de 40 años, resultando una población definitiva de 459 pacientes ( 194 hombres y 265 mujeres). En caso de sospecha de lesión, se estudió la historia clínica de dichos pacientes para determinar la presencia de otros factores de riesgo relacionados con la aterosclerosis, y se hizo una valoración aproximada de la densidad ósea de las mismas. Resultados: 13 individuos (2,83\% de la población de estudio), (53,84\% hombres y $46,15 \%$ mujeres) presentaron radiopacidades en las ortopantomografías y telerradiografías que fueron clasificadas como placas de ateroma. Dichas lesiones, medidas en una escala de grises, registraron unos valores muy similares al obtenido a nivel del hueso alveolar retromolar. De los 13 casos positivos, 30,76\% presentaron las lesiones bilateralmente, y $69,23 \%$ unilateralmente. Conclusión: el odontólogo deberá prestar especial atención a la posible detección de placas de ateroma mediante radiografías panorámicas y telerradiografías, especialmente en aquellos pacientes con mayor riesgo de stroke.
\end{abstract}

Palabras clave: Enfermedad cerebrovascular, Ortopantomografía, Telerradiografía, Placas de ateroma.

Abstract: Introduction: Stroke is the third leading cause of death in the United States and almost the half of deaths in Spain. One of the possible causes of stroke is the formation of atheroma plaques in the carotid bifurcation. Eventually, these plaques calcify and can be detected by chance on panoramic and lateral cephalometric radiographs. Material and Methods: The panoramic radiographs of 1,300 patients of the Dental School of the University of Oviedo, Spain, were evaluated. For our study, only patients over the age of 40 were analized, resulting in a final population of 459 patients ( 194 men and 265 women). In the cases in which a lesion was suspected, the dental history of the patients was studied in order to determine the presence of risk factors related with atherosclerosis: tobacco, alcohol, prior stroke or ischemic attacks, hypertension. Results: 13 individuals $(2,83 \%$ of the final study population), (53,84\% men and $46,15 \%$ women) had radiopacities in the panoramic radiographs which were classified as atheroma plaques. Those lesions were measured in a greyscale and the results were very similar to those obtained in the retromolar alveolar bone. $30,76 \%$ of the positive cases, showed bilateral radiopacities, and $69,23 \%$ unilateral ones. Conclusion: dentists must pay special attention to the possible detection of atheroma plaques on panoramic and lateral cephalometric radiographs, specially in those patiens with high risk of stroke.

Key words: Cerebrovascular disorders, Orthopantomography, Teleradiography, Atheroma plaques.

BIBLID [1138-123X (2006)11:3; mayo-junio 265-380]

Senosiain-Oroquieta A, Pardo-López B, de Carlos-Villafranca F, González-Montoto G, Cobo-Plana J. Detección de placas de ateroma mediante radiografías dentales. RCOE 2006;11(3):297-303. 


\section{Introducción}

El accidente cerebrovascular o "stroke» es la tercera causa de muerte en los Estados Unidos, después de la enfermedad cardiovascular y el cáncer. Así, 12 de cada 10.000 americanos padecen un accidente cerebrovascular $^{1 *}$. En España, casi la mitad de las muertes ocurridas cada año, se deben a la ateroesclerosis y es la población mayor de 55 años la que presenta mayor riesgo.

Cualquier enfermedad que afecte a la pared arterial, sobre todo si provoca que su luz se estreche o se obstruya, puede dar lugar a que disminuya o se bloquee del todo el flujo de sangre que llega a través de ella a los tejidos. Si esto sucede, los tejidos recibirán un aporte de sangre y de oxígeno insuficiente, produciéndose en ellos una lesión, lo que alterará su funcionamiento.

La ateroesclerosis es una enfermedad caracterizada por el desarrollo de múltiples lesiones focales Ilamadas placas de ateroma en la pared arterial. Estas placas están compuestas por lípidos, como el colesterol, y por células inflamatorias, como los monocitos, que desde la sangre penetran en el interior. Se sabe que existen diversos factores de riesgo, como la hipertensión, hipercolesterolemia y tabaquismo, que pueden dañar el endotelio. Además de estos, la diabetes mellitus, la obesidad y la menopausia, contribuyen al desarrollo de esta patología ${ }^{2}$. En ocasiones, estas placas se calcifican, pudiéndo entonces ser detectadas radiográficamente. Friedlander y Lande ${ }^{3^{*}}$, fueron los primeros en describir la presencia de calcificaciones en el área de la arteria carótida mediante radiografías panorámicas realizadas para el diagnóstico dental de rutina, y encontraron una prevalencia de aproximadamente $2 \%$ en una muestra de 1000 pacientes de edad avanzada.

La ateroesclerosis puede tardar mucho tiempo en dar manifestaciones clínicas, o incluso no llegar a producirlas nunca. Por ello, resulta relevante detectarla precozmente antes de que las placas de ateroma se compliquen. Con este fin, es preciso realizar chequeos vasculares a partir de la tercera década de vida, especialmente en personas con antecedente familiares, valorando también los posibles factores de riesgo citados. Actualmente, mediante analítica se evalúa la presencia de determinados marcadores de daño vascular que pueden dar la primera señal de alarma, pero son las técnicas de imagen vascular (ecografía doppler y resonancia magnética nuclear), las que aportan la mayor fiabilidad diagnóstica.

Las placas de ateroma aparecen a la sombra de la faringe a nivel del área de la bifurcación carotídea. Estas lesiones aparecen parcialmente calcificadas y por tanto son visibles en las radiografías de la cara y el cuello, así como en las radiografías anteroposteriores de la columna cervical, del cráneo, huesos faciales, tórax ${ }^{4,5}$

Mediante el presente estudio, pretendemos constatar el papel que puede tener el dentista tras una exploración rutinaria odontológica, como son las radiografías panorámicas, en la detección de las placas de ateroma depositadas en la bifurcación carotídea, y que pueden ser responsables de una gran parte de los accidentes cerebrovasculares.

\section{Material 4 mérodo}

Para llevar a cabo nuestro estudio, se realizó una revisión de una población de riesgo sobre 1300 ortopantomografías del archivo de la Escuela de Estomatología Clínica Universitaria de Odontología, de la Facultad de Medicina de la Universidad de Oviedo. De la población inicial solamente se consideraron los pacientes que tenían una edad superior a 40 años, resultando así una población final de estudio de 459 pacientes (194 hombres y 265 mujeres).

En los casos considerados como positivos, se estudió más exhaustivamente la historia clínica del paciente, con el fin de relacionar la presencia de las lesiones con otros factores de riesgo como la hipertensión, hipercolesterolemia, antecedentes cardíacos o el tabaco. En estos pacientes se repitió el registro radiográfico, y en caso de duda razonable, se realizó una telerradiografía lateral de cráneo como comprobante.

Mediante el programa de análisis de imagen Leica Qwin Pro (Leica Microsystem Imaging Solutions Ltd, United Kingdom) se valoró la densidad ósea aproximada de las supuestas lesiones utilizando para ello una escala de grises con valores que oscilaban entre 0 (negro) y 255 (blanco), comparándose los valores obtenidos con los registrados a nivel del hueso alveolar retromolar y de la vía aérea pericarotídea.

\section{Resultados}

13 pacientes (7 hombres, con edades entre 44-63 años, y 6 mujeres, 


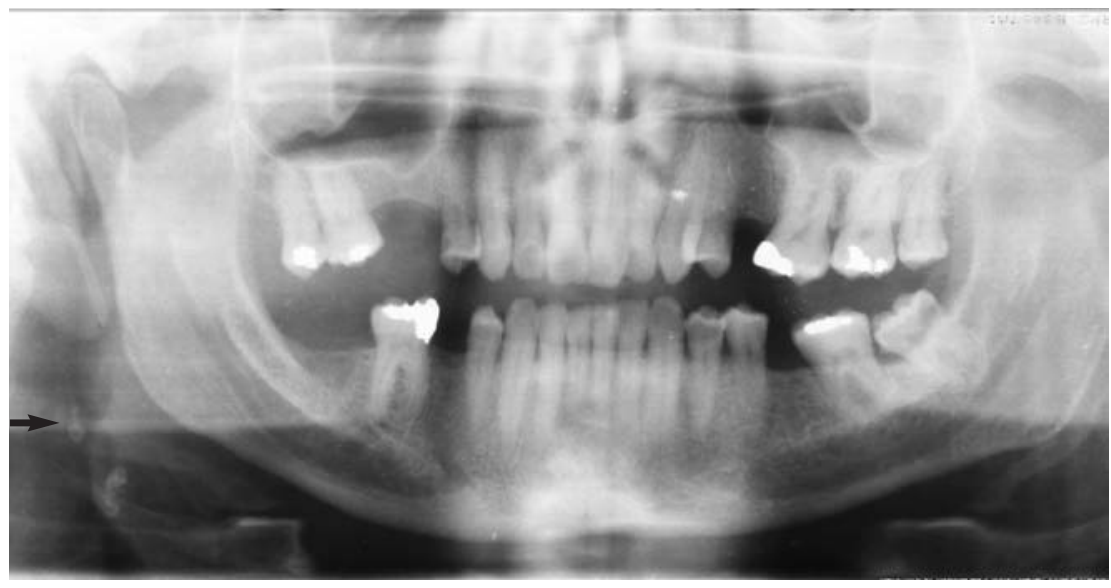

Figura 1. ortopantomografía con placas de ateroma en el área descrita.

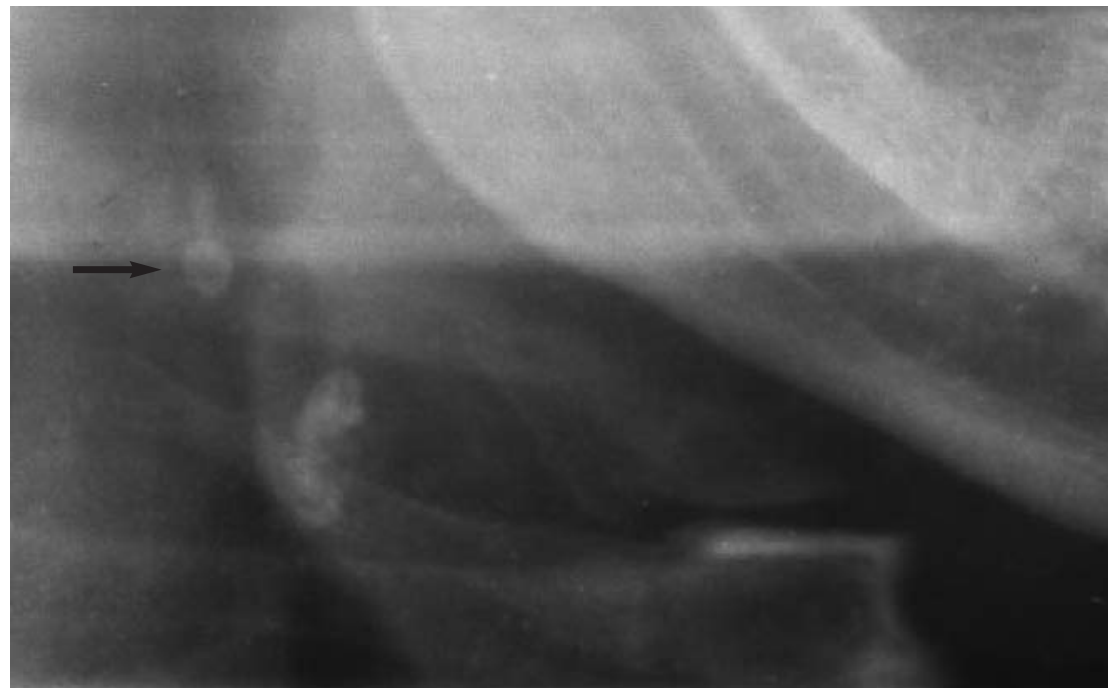

Figura 2. Ortopantomografia con placas de ateroma a mayor aumento.

con edades entre 41-81 años) presentaron radiopacidades en las ortopantomografías y telerradiografías que fueron clasificadas como posibles placas de ateroma (figs. 1,2,3). Esta muestra viene a representar $2,83 \%$ de la población estudiada. De los 13 posibles casos detectados, en el 30,76\% de ellos, las lesiones aparecían bilateralmente, mientras que en el 69,23\% restantes se presentaron de forma unilateral. El aspecto radiológico de las lesiones, masas nodulares hetero- géneas, coincide con el descrito en investigaciones anteriores.

Ninguno de los pacientes refería haber sufrido un accidente cerebrovascular. El 30,76\% de los casos positivos, refirieron estar en tratamiento de hipertensión arterial, el 15,38\% recibían antidiabéticos orales, y el 7,69\% manifestó padecer alteraciones de la coagulación tras haber recibido radioterapia. Un hecho importante fue que, el $38,46 \%$ de los 13 pacientes, reconocían ser fumadores de, al menos, diez cigarrillos al día. Mientras que el valor medio en la escala de grises a nivel de la vía aérea pericarotídea fue de 57,02, el obtenido para las placas de ateroma $(106,34)$ resultó similar al registrado en el hueso alveolar retromolar $(130,84)$.

\section{Discusión}

La formación de placas de ateroma se inicia en casi todos los individuos en la primera o segunda década de vida, de forma que puede empezar a producir manifestaciones clínicas en la tercera o cuarta década, acrecentándose su prevalencia de forma exponencial a partir de la quinta década y pasa a alcanzar un impacto sanitario máximo a partir de esta etapa.

Las placas de ateroma van aumentando con los años lenta y paulatinamente en tamaño y grosor a medida que se acumulan depósitos de grasa y células en su interior, ocupando la luz arterial y con el consiguiente estrechamiento de la arteria.

Las placas de ateroma, radiológicamente, aparecen como masas nodulares heterogéneas y radiopacas o como líneas verticales también radiopacas, por la calcificación de las mismas, siguiendo el trayecto del vaso. La localización suele ser posteroinferior al ángulo de la mandíbula, aproximadamentea a nivel del margen inferior de la tercera vértebra cervical (entre C3 y C4) a nivel o por debajo del hueso hioides (fig. 4). A la hora de realizar el diagnóstico diferencial hay que tener en cuenta otras entidades anatómicas y patológicas, tales como las que se muestran en las tablas 1 y 2 . 


\begin{tabular}{|c|c|}
\hline $\begin{array}{r}\text { Tabla 1.Princinales } \\
\text { esłableces }\end{array}$ & $\begin{array}{l}\text { des anatómicas con las que deberá } \\
\text { diagnóstico diferencialb-8** }\end{array}$ \\
\hline $\begin{array}{l}\text { Entidad anatómica } \\
\text { Hueso Hioides }\end{array}$ & Descripción \\
\hline Proceso estiloides & $\begin{array}{l}\text { Estructura bilateral que se origina a partir del } \\
\text { hueso temporal. En la radiografía aparece en- } \\
\text { tre la rama mandibular y la apófisis mastoides }\end{array}$ \\
\hline $\begin{array}{l}\text { Ligamento estilomandibular } \\
\text { calcificado }\end{array}$ & $\begin{array}{l}\text { Desde la parte más inferior de la apófisis esti- } \\
\text { loides hasta el ángulo mandibular }\end{array}$ \\
\hline Ligamento estilohioideo & $\begin{array}{l}\text { Desciende desde la apófisis estiloides y conec- } \\
\text { ta con el asta menor del hueso hioides. Puede } \\
\text { aparecer calcificado, continuo o segmentado } \\
\text { (Síndrome Eagle) }\end{array}$ \\
\hline Cartílago tiroides & El principal cartílago de la laringe \\
\hline Cartílago triqueon & $\begin{array}{l}\text { Si está calcificado, se aprecia una radiopacidad } \\
\text { de tamaño de grano de trigo, justo debajo del } \\
\text { asta mayor del hioides }\end{array}$ \\
\hline Epiglotis & Por encima del asta mayor del hioides \\
\hline Paladar blando & \\
\hline Lengua & \\
\hline Tubérculo anterior del Atlas & \\
\hline Lóbulo de la oreja & $\begin{array}{l}\text { Aparece como una sombra de radiolucidez } \\
\text { media por detrás de la rama mandibular }\end{array}$ \\
\hline
\end{tabular}

\begin{tabular}{|ll|}
\hline \multicolumn{2}{|c|}{ Tabla e. Principales entidades patológicas con las que deberá } \\
esłablecerse un diagnóstico diferencial
\end{tabular}

Algunas de las entidades que pueden inducir a error con más frecuencia son los nódulos linfáticos calcifica- dos y los sialolitos. Para establecer un diagnóstico diferencial, se deberá proceder a una exploración rutinaria del cuello del paciente, así como de las glándulas salivares. Los tonsiolitos, sialolitos de la submandibular y los nódulos linfáticos calcificados son casi siempre palpables. Sin embargo, estos últimos pueden aparecer enmascarados por el músculo esternocleidomastoideo, de manera que, para localizarlos, el clínico debe de realizar una palpación cuidadosa entre el borde anterior y posterior de dicho músculo. Por el contrario, las placas de ateroma, nunca son palpables y se encuentran internas al músculo esternocleidomastoideo y la pared de la carótida.

Para el diagnóstico de los sialolitos, también se utiliza la técnica de palpación bimanual: se sitúa una mano sobre el borde inferior o ángulo mandibula y la glándula debe ser desplazada medial, posterior y superiormente. El dedo índice de la otra mano se sitúa lo más posterior en el surco lingual (en el borde del músculo milohioideo), y mientras comprimimos la glándula, debe percibirse el sialolito.

El hallazgo de las placas de ateroma mediante radiografías, se trata de algo totalmente casual y el paciente no suele presentar ninguna sintomatología. Ante una imagen sospechosa en un paciente en población de riesgo, el dentista deberá remitir al paciente a su especialista.

El mejor tratamiento de la ateroesclerosis es su prevención primaria. Los principales métodos diagnósticos son los estudios doppler de ultrasonidos y la angiografía de sustracción digital, de menor utilización por los inconvenientes que presenta ${ }^{9}$. Doris et $\mathrm{al}^{10}$ encontraron una asociación estadísticamente significativa entre el grado de calcificación aparente en la radiografía y el grado de severidad 

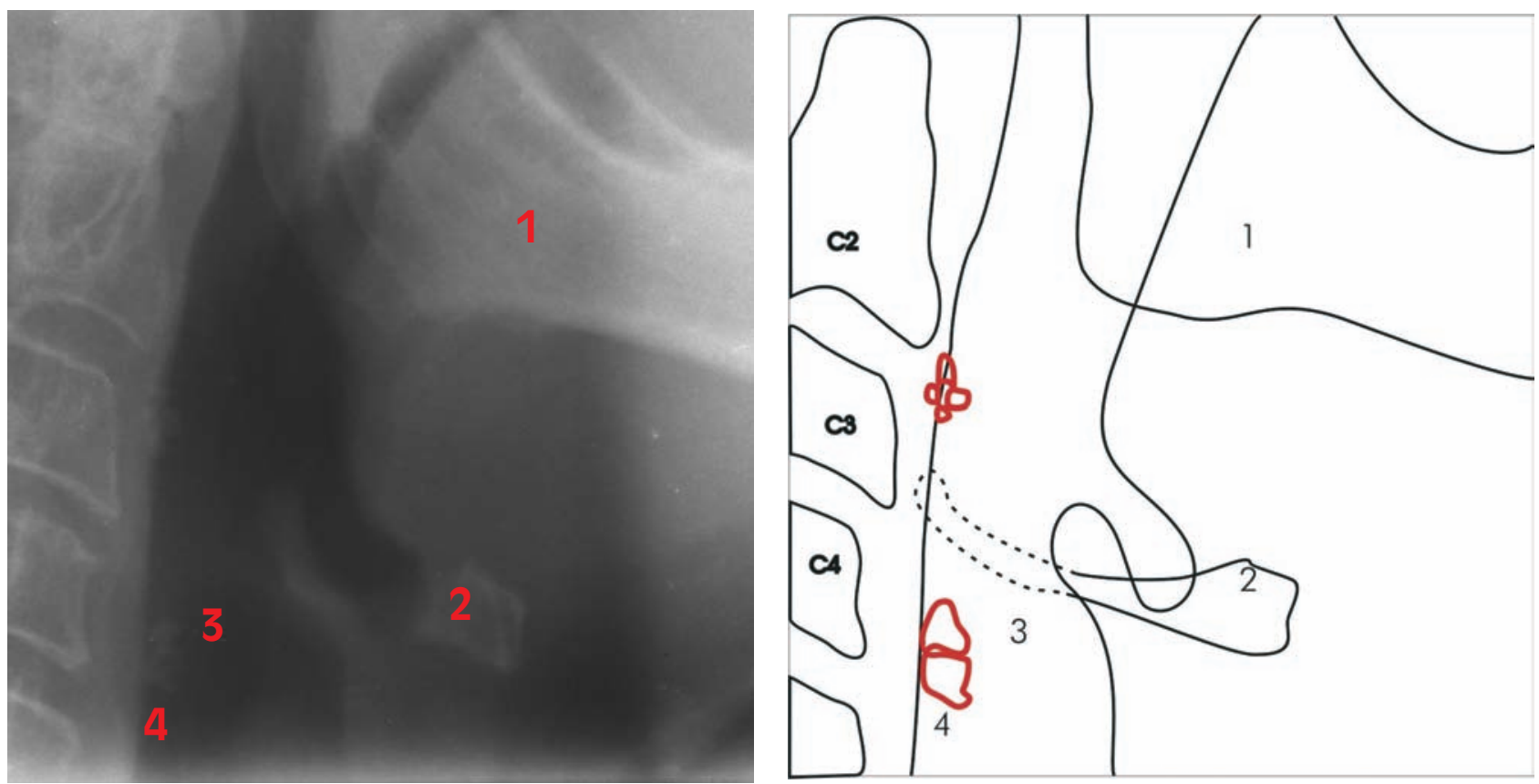

Figuras 3 y 4. 1: ángulo mandibular, 2: hueso hioides, 3: vía aérea pericarotídea, 4: placas de ateroma.

de aterosclerosis demostrado a través de la angiografía de sustracción digital.

Friedlander y Barton (1994), publicaron un estudio en el que demostraron mediante termografía electrónica que en aquellos casos de mayor obstrucción de la luz arterial, el flujo sanguíneo y en consecuencia la perfusión, se va a ver alterado de tal manera que va a resultar en una menor temperatura corporal de los tejidos correspondientes.

Una de las limitaciones de las radiografías panorámicas en la detección de las placas de ateroma, es que la localización de la bifurcación carotídea, donde normalmente asientan dichas placas, es variable en sentido craneo-caudal, pudiendo quedar, en ocasiones, fuera del alcance de dicho tipo de radiografía.

Diversos estudios científicos parecen confirmar que aquellos pacientes con cáncer de cabeza y cuello, los cuales han sido sometidos a radioterapia (40-72 Gy), además de la ya conocida osteorradionecrosis, tienen mayor probabilidad de desarrollar placas de ateroma en la bifurcación carotídea. Dichas lesiones no parecen detectarse de manera inmediata, sino a partir de los tres años posteriores al período postirradiación ${ }^{11,12}$. Según Friedlander ${ }^{13^{*}}$, la diferencia es estadísticamente significativa $(p=0,001)$ entre los indivios sometidos a radioterapia $(27,9 \%)$ y aquellos individuos de características similares, pero que no han recibido radioterapia (4,9\%).

La patogenia exacta del proceso se desconoce, pero diversos estudios describen las diferencias hemodinámicas en aproximadamente el $25 \%$ de los pacientes sometidos a radioterapia $^{14,15}$. Todos estos cambios hemodinámicos conducen a la obstrucción del fluido sanguíneo.
Los enfermos de diabetes tipo ॥ tienen mayor probabilidad de sufrir un colapso circulatorio debido al estado de hiperglucemia, hiperlipidemia e hipertensión que presentan ${ }^{16-19^{*}}$. Friedlander y Maeder (2000), observaron en un estudio sobre 49 pacientes (con edad media de 66,2 años) varones con diabetes tipo II, que el $20,4 \%$ presentaban placas de ateroma detectables en la radiogradía. Por el contrario, la prevalencia en el grupo control era tan sólo del 4\%. Otros autores, hablan de una prevalencia de 2-4 veces mayor en los pacientes diabéticos ${ }^{20-23}$. La explicación a estos resultados reside en que los sujetos afectos de diabetes tipo II, especialmente en aquellos casos que no tienen un buen control de la enfermedad, se ven afectados por enfermedades a nivel micro y macrovascular. De hecho, las alteraciones a nivel de los grandes vasos es la principal causa de morbilidad y 
mortalidad en los pacientes de diabetes tipo II. Los principales vasos afectados son las arterias coronarias (angina, infarto de miocardio), vasos de la extremidad inferior (gangrena) y la arteria carótida (stroke) $)^{24}$.

Diversos estudios confirman que los enfermos de OSAS (síndrome de apnea obstructiva del sueño) sufren accidentes cerebrovasculares en un porcentaje 3-6 veces mayor respecto a la población normal. La justificación exacta a este hecho se desconoce, pero la formación de placas de ateroma se ha sugerido como una posible causa. Friedlander ${ }^{25^{+}-27}$ ha publicado diversos artículos al respecto. En un estudio realizado sobre 47 varones (con edad media de 59,2 años) enfermos de OSAS, encontró que el 21,3\% de estos pacientes presentaban placas de ateroma, mientras que en el grupo control la prevalencia fue tan solo del $2,5 \%$ ( $p=0,000001)$.

Los episodios de apnea/hipoapnea van seguidos de una hipoxia que va a causar una alteración del sistema nervisoso central. Este estímulo induce a la liberación de catecolaminas que van a aumentar la actividad simpática, y en consecuencia, un aumento transitorio de la presión arterial. La consecuencia de la hipertensión va a ser la disrupción en la integridad del endotelio de los vasos, alterando su permeabilidad.

Friedlander y Altman ${ }^{28,29}$ encontraron en 52 mujeres postmenopáusicas, que el $31 \%$ presenta ateromas detectables en una radiografía panorámica.

Parece, pues, clara la relación entre la formación de placas de ateroma y determinadas enfermedades. Sin embargo, un hecho importante que debemos de tener presente a la hora de interpretar todos estos resultados es que, la mayoría de los individuos de estas muestras reúnen múltiples factores de riesgo para el desarrollo de aterosclerosis.

\section{Conclusión}

El hallazgo de placas de ateroma durante una exploración odontológica rutinaria ha de tenerse en cuenta a la hora de realizar la lectura de una radiografía de ámbito dental (panorámica o telerradiografía).

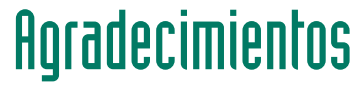

Al servicio de Proceso de Imágenes de los Servicios Científico-Técnicos de la Universidad de Oviedo.

\section{Bibliografia recomendada}

Para profundizar en la lectura de este tema, el/los autor/es considera/an interesantes los artículos que aparecen señalados del siguiente modo: *de interés ${ }^{*}$ de especial interés.

1. *Friedlander AH, Manesh F, Wasterlain CG. Prevalence of detectable carotid artery calcifications on panoramic radiographs of recent stroke victims. Oral Surg Oral Med Oral Pathol. 1990; 77:669-73.

Explicación muy sencilla de la fisiología de formación de las placas de ateroma. Incluye un esquema de la localización de las placas de ateroma en la bifurcación carotídea.

2. Friedlander AH, Friedlander IK. Panoramic dental radiography: an aid in detecting individuals prone to stroke. $\mathrm{Br}$ Dent $\mathrm{J}$. 1996; 181:23-26

3*. Friedlander AH, Lande A. Panoramic radiographic identification of carotid arterial plaques. Oral Surg Oral Med Oral Pathol. 1991:52:102-4

Perfil del paciente con predisposición a presentar placas de ateroma calcificadas en la bifurcación carotídea. Incluye otras pruebas diagnósticas que deben ser pedidas al paciente en caso de posible sospecha.

4. Friedlander AH, Dounis G, Gratt BM. Lateral cephalometric radiographs: an aid in detecting patients at risk of stroke. J Am Dent Assoc. 1996;127:1745-50

5. Friedlander AH. Identification of stroke- prone patients by panoramic and cervical spine radiography. Dentomaxillofac Radiol. 1995; 24:160-4

6**. Friedlander AH. Panoramic radiographs: The differential diagnosis of carotid artery atheromas. Spec Care Dentist. 1995;15:2237.

Establece un claro diagnóstico diferencial de las placas de ateroma calcificadas con otras entidades anatómicas y patológicas.

7. Carter LC. Discrimination between calcified triticeous cartilage and calcified carotid atheroma on panoramic radiography. Oral Surg Oral Med Oral Pathol Oral Radiol Endod. 2000;90:108-10.

8. Almog DM, Tsimidis K, Moss ME, et al. Evaluation of a training program for detection of carotid artery calcifications on panoramic radiographs. Oral Surg Oral Med Oral Pathol Oral Radiol Endod. 2000;90:111-7.

9. Friedlander AH, Gratt BM. Panoramic Dental Radiography as an Aid in detecting Patients at Risk for Stroke. J Oral Maxillofac Surg. 1994;52:1257-62.

10. Doris I, Dobranowski J, Franchetto AA, et al. The relevance of Detectin Carotid Artery Calcification on Plain Radiograph. Stroke. 1993;24:1330-4.

11. Cugini G, Sciacero P, Geda G, et al. Danno indotto sui grossi vasi del collo da irradiazo- 
ne del distretto ORL: studio mediante ecoDoppler. Radiol Med. 1990;80(1-2):56-62.

12. Semergidis T, Vairaktaris E, Iatrou I, et al. Blood suply to the oral and maxillofacial tisúes following radiation therapy: a prospective ultrasonographic study. J Cranio-Maxillofac Surg. 1996;24:16-23.

13*. Friedlander AH, Eichstaedt RM, Friedlander IK, et al. Detection of Radiation-Induced, Accelerated Atherosclerosis in Patients With Osteoradionecrosis by Panoramic Radiography. J Oral Maxillofac Surg. 1998;56:455-9.

Demuestra estadísticamente la mayor predisposición que presentan los pacientes sometidos a radioterapia de cabeza y cuello de padecer un colapso circulatorio.

14. Scholz E, Diener HC, Voss AC. Vascular alteration of extracranial arteries after radiotherapy of tumors of the head and neck. Strahlentherapic. 1982;158:290-7.

15. Elerding SC, Fernandez RN, Grotta JC, et al. Carotid artery disease following external cervical irradiation. Ann Surg. 1981; 194:609-15.

16. Wingo PA, Tong T, Bolden S. Cancer statistics, 1995. CA Cancer J Clin. 1995; 45:8.

17. Boring CC, Squires TS, Tong T. Cancer statistics, 1992. CA Cancer J Clin 1992;42:19-38.

18. Vokes EE, Weichselbaum RR, Lippman SM,et al. Head and neck cancer. N Engl J Med. 1993;328:184-94.
19*. Friedlander AH, Maeder LA. The prevalence of calcified carotid artery atheromas on the panoramic radiographs of patients with 2 types diabetes mellitus. Oral Surg Oral Med Oral Pathol Oral Radiol Endod. 2000;89:420424.

Explica los motives por los cuales en los pacientes con diabetes melliturs tipo II existe una mayor probabilidad de encontrar placas de ateroma calcificadas en la bifurcación carotídea

20. Friedlander AH, Baker JD. Panoramic radiography: an aid in detecting patients at risk of cerebrovascular accident. J Am Dent Assoc 1994;125:1598-1603.

21. Ueta E, Osaki T, Yoneda K, et al. The prevalence of diabetes mellitus in odontogenic infections and oral candidiasis: an analysis of neutrophil suppression. J Oral Pathol Med. 1993;22:168-174.

22. Gibson J, Lamey PJ, Lewis MAO, et al. Oral manifestations of previously undiagnosed non-insulin dependent diabetes mellitus. Oral Pathol Med. 1990;19:284-7.

23. Sonis ST, Fazio RC, Fang L. En: Principles and practice of oral medicine. Philadelphia: WB Sanders Co. 2nd ed, 1995:131-145.

24. Uusitupa MI, Niskanen LK, Siitonen O, et al. Ten year cardiovascular mortality in relation to risk factors and abnormalities in lipoprotein composition in type 2 (non- insulin-dependent) diabetic and non-diabetic subjects. Diabetologia. 1993;36:117584.

25*. Friedlander AH, Yueb R, Littner MR. The prevalence of Calcified Carotid Artery Atheromas in Patients With Obstructive Sleep Apnea Syndrome. J Oral Maxillofac Surg. 1998;56:950-4.

Comenta las rezones fisiológicas que existen para que los pacientes con apnea obstructiva tengan un mayor riesgo de padecer un colapso circulatorio.

26. Friedlander AH, Friedlander IK, Yueh R, et al. The prevalence of Carotid Atheromas Seen on Panoramic Radiographs of Patients With Obstructive Sleep Apnea and Their Relation to Risk Factors for Atherosclerosis. J Oral Maxillofac Surg. 1999;57:516-21.

27. Friedlander AH, Friedlander IK, Pogrel MA. Dentistry's role in the diagnosis and comanagement of patients with sleep apnoea/hypopnoea syndrome. Br Dental J. 2000; 189:76-80.

28. Suárez Cunqueiro MM, Düker J, Schön R, et al. Detección de ateromas en la arteria carótida a través de la radiografía panorámica. Revisión de la bibliografía. Rev Vasca Odontoestomatol 2002;12:12-7.

29. Bonita R, Beaglehole R. Cerebrovascular disease: explaining stroke mortality trones. Lancet. 1993;341:1510-11. 